DEPARTAMENTO DE ANATOMIA PATOLOGICA

Diretor: Prof. Dr. Euclydes Onofre Martins

\title{
CARCINOMAS PULMONARES PRIMITIVOS E METASTÁTICOS, EM CÃES
}

(PRIMARY ANI METASTATIC PULMONARY CARCINOMAS OF THE DOG)

A.vTONio G. Ferri

Assistente

Muitos são os problemas que as ncoplasias pulmonares têm despertado entre os investigadores, destacando-se os referentes à etiologia, histogênese, classificação, nomenclatura e freqüência.

Relativamente à etiologia, ligada que está à própria essência do câncer em geral, pouco ou pràticamente nada se conhece, discutindo-se, entretanto, se inúmeras causas não seriam as responsáveis pelo aparecimento do carcinoma pulmonar. Dentre as múltiplas causas que tèm sido responsabilizadas como determinantes ou pelo menos coadjuvantes na gênese destas neoplasias, destaca-se, em patologia humana, o fumo, segundo Müller (1939) e Doll (1953), entre outros.

A êste problema se prende o do aumento da incidência dos carcinomas primitivos, largamente debatidos em medicina humana, sendo grande o número de autores que acredita haver aumento de incidência, entre os quais cita-se Frubluinc \& HorRenBerger (1952), Weber \& Noll (1952) e Henschen (cit. Monlux - 1952).

Há, entretanto, pesquisadores que pensam ser apenas aparente o aumento de freqüência do carcinoma primitivo do pulmão, como por exemplo RIGDON \& KIRCIIOFF (1951).

Também em patologia veterinária tem esta questāo levantado algumas controvérsias, sendo NiEBERLE \& CoHrs (1949) de opiniāo que os carcinomas pulmonares primitivos no cão, têm aumentado de incidência nos últimos anos, enquanto que outros como JoEsT (1929), JACKSON (1936), Leinati (1948), Gianini (1948), Monari e col. (1949) e Muligan (1949) afĩrmam não só nāo haver maior incidência, como também julgam ser uma neoplasia rara.

Diante das controvérsias existentes, a fim de colaborar para a soJução de tão importante problema, resolvemos colecionar os casos des- 
critos na literatura e também estudar os carcinomas primitivos e secundários arquivados no Departamento de Anatomia Patológica da Faculdade de Medicina Veterinária.

Por outro lado, baseados nas observaçōes aqui apresentadas, sāo abordados sucintamente, além da freqüência, os problemas relativos à histogênese, nomenclatura e classificação dos carcinomas primitivos do pulmão.

\section{MATERIAL E METODOS}

No curso dos trabalhos de rotina, foram necropsiados 1.200 cầes, até o ano de 1953 inclusive, que estão registrados nos arquivos dêste Departamento. Êstes animais, de várias procedências, de ambos os sexos, das mais variadas raças, predominando, entretanto, os cães sem raça definida, eram de idade variando de 1 dia a 18 anos. Deve acrescentar-se que uma parte dêstes animais foi sacrificada ou por ser portadora de moléstias incuráveis ou para demonstração de técnica de necroscopia.

Durante estas necroscopias foram coletados 32 carcinomas do pulmão, que depois de fixados em formol neutro a $10 \%$, foram incluídos em parafina e corados pela hematoxilina-eosina. Além disso, sempre que se tornou necessário para elucidação de diagnóstico, foram feitas coloraçōes eletivas pelos métodos de van Gieson, Mallory, Foot-Wilder, Bielschowsky e orceina ácida.

Por outro lado, foram colhidos da literatura possivel de ser compulsada, todos os demais dados que se fizeram necessários, para as verificaçōes de freqüência dos tumores primitivos do pulmão, nos animais.

\section{RESULTADOS E DISCUSSAO}

Incidência - Em minuciosa pesquisa bibliográfica, foi possivel verificar que até o presente foram descritos 156 casos de tumores pulmonares primários, nos animais domésticos, os quais se acham distribuídos por espécie, na tabela I.

Os dados fornecidos pela tabela I mostram que os tumores pulmonares, em geral, são raros nos animais. Deve salientar-se que os dados relativos aos tumores dos ovinos devem ser tomados com precauçāo, uma vez que alguns dos tumores aqui incluídos referem-se provàvelmente à adenomatose pulmonar dos ovinos, descrita por JoHNE (1891), 
Tabela I - Relação dos tumores pulmonares nos animais domésticos.

\begin{tabular}{c|c}
\hline \multicolumn{1}{c|}{ A n i m a i s } & $N^{\bullet}$ de casos \\
\hline \hline Cães $\ldots \ldots \ldots \ldots \ldots \ldots$ & 71 \\
Gatos $\ldots \ldots \ldots \ldots \ldots \ldots$ & 8 \\
Eqüinos $\ldots \ldots \ldots \ldots \ldots \ldots$ & 33 \\
Bovinos $\ldots \ldots \ldots \ldots \ldots \ldots$ & 32 \\
Ovinos $\ldots \ldots \ldots \ldots \ldots$ & 12 \\
Total $\ldots \ldots \ldots \ldots \ldots$ & 156 \\
\hline
\end{tabular}

COWDRY \& MARSH (1927), a qual tem sido interpretada como um processo reativo e não tumoral. Entretanto, Cuba Caparó (1954) verificou em algumas destas lesões, uma transformação carcinomatosa com metástase nos gânglios linfáticos regionais. Estes fatos tornam extremamente difícil, pois, uma análise da literatura, com relação aos tumores pulmonares dêstes animais.

Nota-se ainda do exame dessa tabela, que são os căes os mais freqüentemente afetados pelos tumores, e que até a presente data não foram descritos blastomas em pulmão de suinos.

A maior freqüência nos cães pode ser explicada por ser êste animal sujeito a constantes inalações irritativas pelo seu "habitat" citadino, o que encontraria paralelo em relação à patologia humana.

Por outro lado, a ausência dêstes tumores em suinos tem sua explicação em seu curto periodo de vida, por serem abatidos com pouca idade (antes de 2 anos, em geral).

No que diz respeito exclusivamente aos carcinomas primitivos nos animais, a tabela II resume os dados encontrados na pesquisa bibliogräfica.

Do confronto das tabelas $I$ e $\Pi$, verifica-se que os carcinomas são muito mais freqüentes que os demais tumores em todos os animais, porém, no cão prảticamente todos os tumores observados são carcinomas, com exceção de um condroma observado por NoCARD (cit. KITT, 1923) e um histiocitoma descrito por FERri \& TAUSK (1955). O lipoma múltiplo, descrito por SEMMER (1889), com tôda probabilidade não se tratava de neoformação tumoral. 


\begin{tabular}{|c|c|}
\hline Animais & $\mathbf{N}^{\circ}$ de casos \\
\hline$=-1=0=1$ & $\frac{1}{1}=-=$ \\
\hline Cão $\ldots . . . \ldots \ldots \ldots$ & 69 \\
\hline Gato & 6 \\
\hline Equïino .. & 27 \\
\hline Bovino ....... & 28 \\
\hline Ovino .. & 6 \\
\hline Total & 136 \\
\hline
\end{tabular}

Com referência ao aumento de incidência dos carcinomas pulmonares primitivos do cão, a tabela III reune os dados da literatura, grupando-os por espaços de 20 anos.

Tabela III - Incidéncia dos carcinomas primitivos do pulmão, em cãcs

\begin{tabular}{|c|c|}
\hline Anos & $N^{D}$ de casos \\
\hline$\because+1-1,-1=$ & $=-\ldots$ \\
\hline $1880-1900 \ldots \ldots \ldots \ldots$ & 8 \\
\hline $1901-1920 \ldots \ldots \ldots \ldots$ & 11 \\
\hline $1921-1940 \ldots$ & 26 \\
\hline $1941-1955$ & 24 \\
\hline Total ... & 69 \\
\hline
\end{tabular}

Verifica-se, do exame da tabela III, que nos últimos anos tem havido aumento de incidência dos carcinomas primitivos do pulmāo nos cães, o que estaria de acôrdo com a observaçāo de Nieberle \& CoHrs. Entretanto, segundo alguns autores, êste aumento é apenas o resultado do incremento da pesquisa nos últimos anos.

Os dados, ao que parece, são até a presente data insuficientes para permitirem conclusão definitiva. Esta é, aliás, também a opiniāo de Monlux. 
Do arquivo dêste Departamento foram coletados 32 carcinomas do pulmão, entre primários e metastáticos, em 1.200 necroscopias, os quais sāo apresentados nas tabelas IV e $V$, com as respectivas porcentagens de freqüēncia.

Tabela IV - Freqüência dos carcinomas primitivos e metastáticos $\mathrm{cm} 1.200$ necroscopias.

\begin{tabular}{c|c|c}
\hline Discriminaçāo & No de casos & Porcentagem \\
\hline Ca. primitivos ....... & 4 & 0,33 \\
Ca. metastáticos ..... & 28 & 2,33 \\
Total ........... & 32 & 2,66 \\
\hline
\end{tabular}

Tabela V -- Rclaçãa dos carcinomas primitivos e metastáticos do pulmão cm cães.

\begin{tabular}{|c|c|c|}
\hline D iscriminação & $\mathbf{N}^{\circ}$ de casos & Porcentagem \\
\hline Ca. primitivo do pulmão $\ldots \ldots \ldots \ldots \ldots \ldots \ldots$ & 4 & 12,50 \\
\hline Ca. metastático da tireóide $\ldots \ldots \ldots \ldots \ldots \ldots$ & 12 & 37,50 \\
\hline Ca. metastático da mama $\ldots \ldots \ldots \ldots \ldots \ldots$ & 10 & 21,25 \\
\hline Ca. metastático do figado $\ldots \ldots \ldots \ldots \ldots \ldots$ & $\mathbf{3}$ & 9,37 \\
\hline Ca. metastático da pele $\ldots \ldots \ldots \ldots \ldots \ldots \ldots$ & 2 & 6,25 \\
\hline Ca. metastático do testiculo $\ldots \ldots \ldots \ldots \ldots \ldots$ & 1 & 3,12 \\
\hline Total $\ldots \ldots \ldots \ldots \ldots \ldots \ldots \ldots \ldots \ldots \ldots \ldots \ldots$ & 32 & - \\
\hline
\end{tabular}

Examinando-se as tabelas IV e $\mathrm{V}$ verifica-se que os carcinomas pulmonares primitivos são processos raros, enquanto que os carcinomas metastáticos são muito mais freqüentes. Êstes dados estão de acôrdo com a antiga observaçāo de Virchow, segundo a qual os órgāos que são sede freqüente de metástases, raramente apresentam tumores primitivos.

Com referência aos carcinomas primitivos do pulmão, STICKeR (1902), em 766 carcinomas de cão, verificou que êstes constituiam $1,30 \%$ dos casos.

GUERrini (1908) cita as estatisticas de JoHNe \& CASPER, onde se verifica 4 e $5 \%$, respectivamente, de porcentagem de incidência no pulmão de cão. 
DOBBERSTEIN (1937), estudando os carcinomas primitivos, concluiu que em tôdas as espécies a freqüência é aproximadamente a mesma. Para o cão, a porcentagem encontrada foi de $4,11 \%$.

Os tumores metastáticos do pulmão, eram provenientes de diferentes tumores de vários órgãos e se acham distribuídos segundo sua freqüência, na tabela VI.

Tabela VI - Freqüência das metástases pulmonares, em cāes.

\begin{tabular}{|c|c|c|c|}
\hline Orgãos & $\begin{array}{l}\text { Carcinomas } \\
\text { primitivos }\end{array}$ & $\begin{array}{c}\text { Metástases no } \\
\text { pulmão }\end{array}$ & $\begin{array}{c}\text { Porcentagem das } \\
\text { metástases }\end{array}$ \\
\hline Tireóide $\ldots \ldots \ldots \ldots \ldots$ & 23 & 12 & 52,17 \\
\hline Mama $\ldots \ldots \ldots \ldots \ldots \ldots$ & 25 & 10 & 40,00 \\
\hline Figado $\ldots \ldots \ldots \ldots$ & 8 & 3 & 37,50 \\
\hline Pele $\ldots . . \ldots \ldots \ldots \ldots$ & 18 & 2 & 11,11 \\
\hline Testículo $\ldots \ldots \ldots \ldots \ldots$ & 10 & 1 & 10,00 \\
\hline Total $\ldots \ldots \ldots \ldots$ & 84 & 28 & 33,33 \\
\hline
\end{tabular}

Pode observar-se do exame da tabela VI, que os carcinomas da tireóide são os que mais vêzes dão metástases no pulmão, seguidos de perto pelos de mama, enquanto que os tumores de pele são os que rarìssimamente dāo metástases no pulmão.

Nos casos aqui estudados, apenas duas vêzes se observou metástase no pulmão, convindo salientar que um dos casos era um mclanocarcinoma com metástases generalizadas e o outro, um carcinoma planocelular corneificado, havendo no pulmão, neste caso, poucos nódulos $\mathrm{e}$ bem circunscritos. E interessante frisar, também, que no cão os tumores da tireóide e da mama dāo metástases muito mais freqüentemente no pulmão que em qualquer outro órgão; assim, em dois casos, foi observado metástase de tumor da tireỏide sỏmente no gânglio linfático mediastinal e três vêzes se verificou metástase de carcinoma da mama no fígado.

Esta observação não concorda com o que se tem verificado $\mathrm{cm}$ patologia humana. Segundo Roussy e col. (1950), as metåstases dos carcinomas da tireóide têm predileção pelo tecido ósseo. Os carcinomas da mama dāo metástases primeiramente nos gânglios linfáticos regionais, vindo o pulmāo e o tecido ósseo em segundo lugar, de acôrdo com DIETRICH (1943). 
Esta diversidade de comportamento em relação ao que tem sido verificado em medicina humana, provàvelmente em parte pode ser explicada pelos recentes estudos de Coman \& LoNG (1951), em relação ao "sistema venoso vertebral". Estes autores, injetando células tumorais na veia safena externa de coelhos e ratos, iam encontrá-las sistemàticamente no pulmāo, entretanto, quando exerciam uma compressāo no abdomen dos animais, as células tumorais atingiam a coluna vertebral.

É provável que a posição do animal dificulte o refluxo do sangue através do "sistema venoso vertebral".

Nos cães, sendo o pulmāo a sede predileta das metástases, particularmente em relação aos neoplasmas da tireóide e da mama, como também do figado, conclui-se que a via hemática é a principal via de propagação dêsses tumores, tendo sido possivel verificar a invasāo de vasos sangüineos e a proliferação a partir de êmbolos tumorais, em muitos casos.

Quanto à idade, os carcinomas primitivos aparecem em média aos 9-10 anos de idade, tendo sido verificado como limite mínimo 14 meses e máximo 17 anos. E quanto ao sexo, foram descritos 22 casos em cães do sexo masculino e 16 em fêmeas. Nos demais casos nāo foram relatados êstes dados.

Sabe-se, além disso, que os carcinomas primitivos ocorrem maior número de vêzes no pulmão direito, tanto em patologia humana como em veterinária, o que se explica por possuir o pulmão direito maior área que o esquerdo. Entretanto, na maioria dos casos os tumores eram bilaterais.

Relativamente aos carcinomas metastáticos, sòmente foram observadas metástases no pulmão, em cães com mais de 7 anos de idade, predominando nos animais de 11-12 anos.

Havia forte predominância em indivíduos do sexo feminino, nāo podendo, entretanto, êste fato ser tomado em consideraçāo, pelo número grande de fêmeas com tumores mamários, incluidos na relação.

Quanto à raça, poucas são as referências e, em geral, os animais nāo apresentavam raça definida.

LESōes.

Carcinomas primitivos - Os carcinomas primitivos no pulmão de cāo têm sido descritos como se apresentando macroscòpicamente como 
nódulos únicos ou múltiplos ou sob forma de massas tumorais infiltrantes, ou ainda, como uma pneumonia carcinomatosa.

Nos casos aqui estudados, êstes aspectos foram observados, sendo que um dêles se apresentava como um foco hepatizado, isto é, uma pneumonia carcinomatosa focal.

Quanto ao aspecto histológico, um dos tumores se mostrava constituido predominantemente por células prismáticas, formando um nódulo bem delimitado do restante do parênquima e mostrando disposiçāo que lembrava a do revestimento brônquico. Havia, entretanto, zonas de indiferenciação acentuada constituida por células arredondadas ou fusiformes. Neste caso foi verificada metástase no encéfalo.

Nos demais casos encontrados, as células se dispunham na maioria das vêzes sob forma de cordões sólidos que se assemelhavam aos brotos carcinomatosos derivados dos epitélios malpighianos, havendo, entretanto, num dos casos, zonas com cẻlulas prismáticas que revestiam os alvéolos. Noutro caso observou-se um dos brônquios com metaplasia epidermóide.

Por êstes aspectos observados, o primeiro dos tumores foi classificado como um carcinoma prismático celular e os demais como carcinomas epidermóides.

Carcinomas metastáticos - Estes se apresentavam como nódulos únicos ou múltiplos de tamanhos os mais variados. Porém, em 2 casos de metástases de carcinoma da tireóide observou-se no pulmão em um dos casos u'a massa tumoral tomando todo o lobo diafragmático. A grande maioria, entretanto, se mostrava constituída por mültiplos nódulos semeados por todo o parênquima pulmonar. Eram os nódulos em geral bem delimitados, muitos dêles fazendo saliência hemisférica na superficie externa e recobertos pela pleura visceral, que muitas vẽzes se mostrava discretamente espessada.

Êste aspecto macroscópico foi observado em todos os casos de tumores metastáticos, quer seja da tireóide, da mama, do figado ou de qualquer outro órgão.

O aspecto histológico lògicamente era variável conforme o tipo de blastoma que deu origem à metástase. Entretanto, de maneira geral verificou-se que o tumor metastático se apresentava com as mesmas caracteristieas que o tumor de origem, mas foi às vêzes observado, entretanto, ser mais anaplástico ou mais diferenciado. 
Em $60 \%$ dos casos estudados não havia diversidade de anaplasia entre o tumor primitivo e o secundário; em $30 \%$ o tumor metastático era menos diferenciado e em $10 \%$ era maior o grau de anaplasia na metástase.

\section{HistogENESE, NOMENCLATURA E CLASSIFICAÇño.}

Por serem questōes interrelacionadas serão abordadas em conjunto.

As classificações existentes têm sido baseadas ou no aspecto morfológico ou na histogênese.

Com relação à histogênese, tem-se discutido muito a origem da céIula da parede alveolar, admitindo uns que seja de origem epitelial, dai o grande número de casos de tumores descritos como carcinomas de células alveolares, enquanto outros afirmam ser a célula alveolar de origem mesenquimal.

A observação de um histiocitoma no pulmāo, por FERRI \& TAUSK, parecendo ter origem nas células da parede alveolar, vem favorecer o ponto de vista de Policard e col. (1944-1947-1950), entre outros. Este assunto, entretanto, já foi abordado em trabalho anterior, razāo pela qual não é aqui discutido pormenorizadamente.

De acôrdo com o ponto de vista estabelecido, todos os carcinomas do pulmão têm origem na árvore brônquica, seja do epitélio de revestimento (carcinomas), seja das glândulas anexas (adenocarcinomas). Boyn (1946) considera os adenocarcinomas originados quer das glândulas anexas quer do epitélio de revestimento - critério seguido por muitos autores, classificando os carcinomas em três grupos: 1) anaplástico ou indiferenciado; 2) adenocarcinoma e 3) carcinoma epidermóide.

Monlux os classifica em: 1) carcinomas de células prismáticas; 2) carcinomas plano-celulares; 3) carcinomas de células mistas e 4) carcinomas indiferenciados.

A denominação de carcinoma epidermóide parece ser mais adequada, razão pela qual foi aqui utilizada. Por outro lado, os carcinomas de células mistas podem perfeitamente ser enquadrados como um subgrupo dos carcinomas indiferenciados. Por estas razões sugere-se a seguinte classificação:

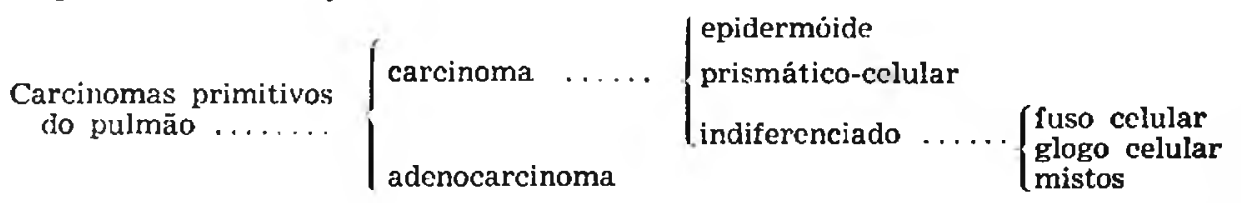


Foram estudados, do ponto de vista de incidência, os carcinomas primitivos do pulmāo, verificados na literatura, acrescentando-se os primários e metastáticos encontrados em 1.200 necroscopias. Foi observada uma freqüência de $0,33 \%$ de carcinomas primitivos e de $2,33 \%$ de metástases no pulmāo, em necroscopia.

Após discussão da questão do aumento de incidência dêsses tumo res, discutiu-se a histogênese, nomenclatura e classificação, apresentando-se pequenas modificaçōes à classificação de MoNLux.

Não é possivel concluir-se sôbre o aumento de incidência em vista do pequeno número de observaçc̄es verificadas na literatura e nas necroscopias realizadas neste Departamento.

\section{SUMMARY AND CONCLUSIONS}

For the interest of reoccurance, a study of the primary pulmonary carcinomas of the dog was made as found in literature, adding the primary and metastatic carcinomas found in 1,200 dog necroscopies. A frequency of $0.33 \%$ primary carcinomas and $2.33 \%$ metastic carcinomas observed in the dog lung was found.

After discussion of the increasing reoccurance problem of these tumors, the histogenesis, nomenclature and classification were discussed bringing up small changes to the MoNLux's classification.

It is not possible to come to a conclusion about the increase problem of incidents because of the small number observed in literature and in the necroscopy realized in this Department.

\section{BIBLIOGPAFIA}

Boyd, W. - 1946 - Compêndio de Patologia Geral c de Anatomia Patológica :406-14. Rio. Guanabara

Coman, D. R. \& LoNG, P. - 1951 - The role of the vertebral venous system in the metastasis of cancer to the spinal column. Experiments with tumor cell suspensions in rats and rabbits. Cancer, $4(3): 610-8$

Cuba Caparaó, A. - 1954 - Adenomatose pulmonar do carneiro. Comunicado à Sociedade Paulista de Patologia em 9-11-54. São Paulo

Cowdry, E. V. \& MARSH, H. - 1927 - Comparative pathology of South African Jagziekte and Montana progressive pneumonia of Shecp. Jour. Exp. Med., $45: 571-8$ 
Dietrich, A. - 1943 - Patologia General y Anatomia Patologica, II :89-91. Barcelona, Francisco Seix

Dobberstein, J. - 1937 - Cit. Monlux

DoLt, R. - 1953 - Bronchial carcinoma : incidence and etiology. Brit. Med. Jour. September :585-90

FerRi, A. G. \& TAUSK, E. - 1955 - Primary pulmonary carcinomas of the dog. Jour. Comp. Path., 65(2):159-67

Fruiling, L. \& Horrenberger, D. - 1952 - De l'augmentation actuclle de la frcquence des cancers d'après la statistique necropsique. Bull. Ass. Fr. Cancer, $\mathbf{3 9}(1): 17-30$

Gianini, G. -- 1948 -.. Sui carcinomi bronco-polmonari ncgli animali domestici. Rev. Anat. Pat. e Oncol., 1(3):1-15

Gijerrini, G. - 1908 - I neoplasmi negli animali. Pathologica, 1(7):156-9

Hensciren, F. - cit. Monlux

JACKson, C. - 1936 - The incidence and pathology of tumours of domesticated animals in South Africa. Onderstep Jour., 6:126-30

Jokst, E. -.. 1929 - Handbuch der speziellen pathologischen Anatomie der Haustiere. 4:808-13. Berlin, Richard Schvetz

JoHNE - 1891 - cit. Monlux

KITT, T. - 1923 - Lehrbuch der pathologischen Anatomie der Haustiere, 2 ;708-15, 5.e Auf., Stuttgart, Ferdinand Enke

Leinsti, L. - 1948 - Anatomia Patologica degli animali domestici. :370-1, 2` ed. Milano. Ambrosiana

Monari, D. - Montroni, L. \& Marcato, A. - 1949 - Anatomia Patologica degli Animali domestici. :197-8. Bologna, Ricardo Pateon

MonluX, W. S. - 1952 - Primary pulmonary neoplasms in domestic animals. Monography published by South-Western, Texas, A. e M. College

Müller, F. H. - 1939 - cit. Doll

Muldigan, R. H. - 1949 - Neoplasms of the dog: 4. Baltimorc, Williams \& Wilkins Co.

Nieberle, K. \& Cohrs, P. - 1949 - Lehrbuch der speziellen pathologischen Anatomia der Haustiere: 170-3. 3.* Auf., Jena, Gustav Fischer

Policaro, A. -- 1947 - Les alignements cellulares dans le poumon. Le problème de la distinction de dcux nature bronchique ou alvéolaire. Bull. Histol. Appl., $24: 127-31$

Policard, A. - 1950 - Sur quelques points du processus de phagocytose par la cellule alveolaire du poumon. Bull. Histol. Appl., $27(3): 1-15$

Policrad, A. \& MUller, F. - 1944 - Sur le development prenatal du poumon et ses facteurs morphogénétiques. Bull. Hist. Appl., 21(1):67-80 
RIGDON, R. H. \& KIRCHoFf, H. - 1951 - Frequency of pulmonary cancer in all malignancies, studied at autopsy. South Med. J., 44:506-12

Roussy, G. - Leroux, R. \& Oberling, C. - 1950 - Précis d'Anatomie Pathologique. :1195-221, 3.e ed. Paris, Masson et Cie.

SEMmer, E. - 1889 - cit. Monlux

StIcker, A. - 1902 - Weber den Kubs der Thiere. Arch. f. klin. chir., 65:616$96 ; 1023-87$

VIRchow, R. L. - cit. Frissel, L. F. \& Kuox, L. C. - 1937 - Primary carcinoma of the lung. A. J. Cancer, $30(2): 219-88$

Weber, K. \& Nolt, G. - 1952 - Uber die Zunahme des Bronchial-carcinoms in Scktions material des Frankfurter Pathologischen Instituts won 1932 bis 1951. Zschr. Krebsforsch, 58(3):364-73 